\title{
Effect of sodium deoxycholate and sodium cholate on DPPC vesicles: A fluorescence anisotropy study with diphenylhexatriene
}

\author{
USHARANI SUBUDDHI and ASHOK K MISHRA* \\ Department of Chemistry, Indian Institute of Technology - Madras, Chennai 600036 \\ e-mail: mishra@iitm.ac.in
}

\begin{abstract}
Effects of two bile salts, namely sodium deoxycholate (NaDC) and sodium cholate (NaC), on DPPC small unilamellar vesicles have been investigated using the steady-state fluorescence anisotropy $\left(r_{s s}\right)$ of diphenylhexatriene $(\mathrm{DPH})$ as a tool. It was found that the variation of $r_{s s}$ is sensitive enough to monitor different stages of interaction of bile salts with DPPC vesicles. NaDC induced significant changes in the membrane well below its CMC $(6 \mathrm{mM})$. Even at $4 \mathrm{mM}$, which is still lower than the CMC, the phospholipids were completely solubilised by the NaDC micelles. The effect of $\mathrm{NaC}$ on DPPC vesicles, however, was much less significant, especially in the sub-micellar concentration regime. Being more hydrophilic $\mathrm{NaC}$ does not interact with the membrane efficiently. Complete solubilisation of phospholipids took place only when the concentration of $\mathrm{NaC}$ was above its CMC (16 $\mathrm{mM})$. The experiments also showed that the bile salt-induced changes of vesicle structure were strongly dependent on the concentration of the bile salt and not on the molar ratio of lipid and bile salt.
\end{abstract}

Keywords. Bile salt; DPPC vesicle; fluorescence anisotropy; 1,6-diphenylhexatriene.

\section{Introduction}

Mixed lipid/detergent systems play an important role in the investigation of membrane properties and functions. ${ }^{1,2}$ Studies of mixtures of micelle-forming bile salts and bilayer-forming phosphatidylcholines are of general interest not only for the understanding of the self-assembly of supramolecular aggregates, ${ }^{3}$ but also for the design of new drug-delivery systems. ${ }^{4,5}$ Progress in the development of highly effective drugs requires the optimisation of existing formulations and the creation of new delivery systems; hence the interest in bile salt/phospholipid mixed micellar systems. These aggregates aid in the solubilisation of poorly soluble drugs as well as in the inclusion of larger drugs. On addition of appropriate sublytic concentrations of bile salt to lipid bilayer, an expansion of the vesicles takes place and momentary defects are induced in liposomal membranes leading to enhanced permeability for molecules up to a size of 70,000 Daltons. This method is known as 'detergent-induced liposome loading' (DILL). ${ }^{6}$ Although their biocompatibility and biodegradability make them safe and efficacious vehicles for medical applications, there still remain several problems to be overcome, one of such pro-

*For correspondence blems being the stability of lipid vesicles in blood and in the gastrointestinal tract. ${ }^{7}$ The stability of liposomes in the gastrointestinal tract is largely affected by the bile salts; hence a proper understanding of the action of bile salts on liposomes is indispensable.

The interaction efficiency of bile salts with phospholipid vesicles depends on a number of factors such as chemical nature and concentration of the bile salt, molecular structure of the lipids, size,
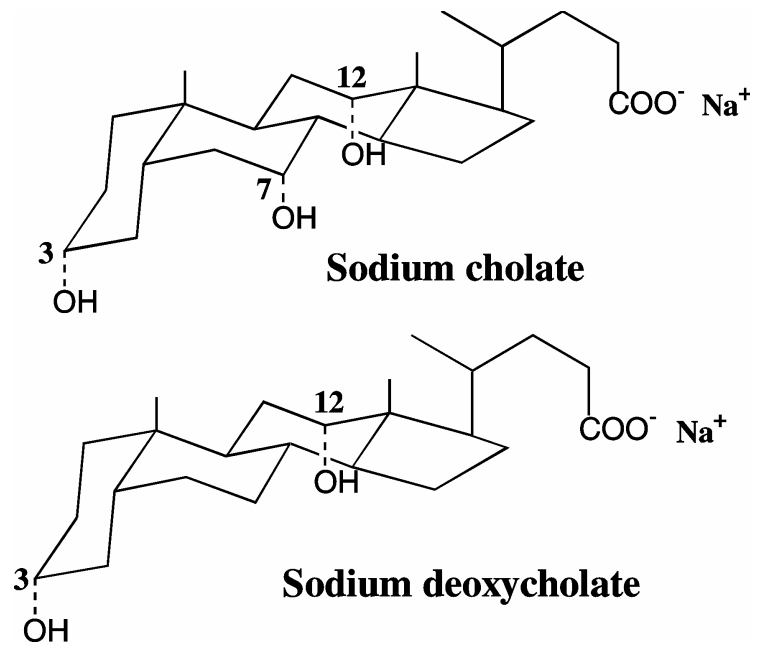

Scheme 1. Molecular structures of sodium cholate $(\mathrm{NaC})$ and sodium deoxycholate $(\mathrm{NaDC})$. 
shape and state of the vesicles, buffer, $\mathrm{pH}$, and temperature ${ }^{8,9}$ Hence, it is desirable to have a clear understanding of the interaction between bile salts and phospholipid vesicles under different experimental conditions and with different kinds of vesicles.

The objective of the present work was to investigate the effect of two bile salts dihydroxy sodium deoxycholate $(\mathrm{NaDC})$ and trihydroxy sodium cholate $(\mathrm{NaC})$ (scheme 1) on dipalmitoylphosphatidylcholine (DPPC) small unilamellar vesicles (SUVs), employing the fluorescence anisotropy of 1,6-diphenylhexatriene (DPH) as a tool.

\section{Experimental}

\subsection{Materials}

Dipalmitoylphosphatidylcholine (DPPC) was purchased from the Sigma Chemical Company (USA) and was used as such. Sodium deoxycholate (NaDC) and sodium cholate $(\mathrm{NaC})$ were obtained from SRL India Ltd. and were recrystallized from hot ethanol. 1,6-diphenylhexatriene (DPH) was purchased from the Sigma Chemical Company (USA) and was used as received. Water, distilled twice from alkaline permanganate solution, was used for the experiments.

\subsection{DPPC vesicle preparation}

For the present work small unilamellar vesicles in the size range $30-50 \mathrm{~nm}$ were used. The vesicles were prepared by ethanol injection method ${ }^{10}$ where an ethanolic solution of the lipid was injected rapidly with the help of a fine needle into water maintained at $50^{\circ} \mathrm{C}$ (optimised condition). The volume of ethanol injected is always less than $1 \% \mathrm{v} / \mathrm{v}$ in order to avoid any damage to the liposome by ethanol.

\subsection{Labelling of vesicles}

Labelling of the vesicles was achieved by adding a measured amount of DPH to the lipid solution before preparation of the liposome, in a molar ratio yielding the desired final lipid/probe ratio, such that $\mathrm{DPH}$ is directly incorporated into the membrane during its formation. For all the experiments, a control solution containing the same concentration of lipid in absence of probe was used as blank. The partition coefficient value $\left(K_{p}\right)$ of DPH in DPPC SUVs is calculated using the equation

$$
F=F_{0} L /\left\{\left(55 \cdot 6 / K_{p}\right)+L\right\}
$$

where $F$ is the fluorescence intensity at $428 \mathrm{~nm}$ at lipid concentration $L, F_{0}$ is the maximum fluorescence resulting from total probe incorporation into membrane. ${ }^{11}$ The $K_{p}$ value is found to be $2 \times 10^{6}$ in the liquid crystalline phase $\left(50^{\circ} \mathrm{C}\right)$. From this value the lipid to probe ratio is calculated to be $\approx 600$ by using $[\mathrm{DPH}]=5 \times 10^{-7} \mathrm{M}$ and $[\mathrm{DPPC}]=3 \times 10^{-4} \mathrm{M}$ and this ratio was maintained for further work.

\subsection{Incorporation of bile salts}

Stock solutions of $\mathrm{NaDC}$ and $\mathrm{NaC}$ were prepared in water. Fresh solutions of bile salt were prepared every time to avoid the problem of aging. The experimental solutions were prepared by adding the desired volume of bile salt stock to the preformed DPPC vesicles and the solution was equilibrated for at least two hours before experiments.

\subsection{Fluorescence measurements}

Fluorescence measurements were carried out with Jobin-Yvon fluorolog II spectrofluorimeter. Excitation and emission spectra were recorded with $5 / 5 \mathrm{~nm}$ slit widths. For temperature dependence experiments the temperature was controlled by circulating water through a jacketted cuvette holder from a refrigerated bath (Insref Ultra Cryostat, India). Temperature was also checked inside the cuvette before and after the experiments; the variation was negligible. The steady-state fluorescence anisotropy $\left(r_{s s}\right)$ values were obtained using the expression

$$
r_{s s}=\left(I \Vdash-G I_{\perp}\right) /\left(I \Vdash+2 G I_{\perp}\right),
$$

where $I \wedge$ and $I_{\perp}$ are fluorescence intensities when the emission polarizer is parallel and perpendicular, respectively, to the direction of polarization of the excitation beam, and $G$ is the factor that corrects for unequal transmission by the diffraction gratings of the instrument for vertically and horizontally polarized light.

\section{Results and discussion}

DPH is one of the most popular fluorescence anisotropy probes for bilayer membranes. ${ }^{12-14}$ It is a rodlike molecule, whose absorption and emission 
dipoles are collinear and lie along the principal molecular axis, which is perpendicular to the membrane plane. The probe is located deep within the hydrophobic core region of the phospholipid bilayer. ${ }^{14}$ Thus any modification in the movement of the chains or packing is sensitively reflected in the fluorescence anisotropy of DPH. Both fluorescence intensity and fluorescence anisotropy of DPH are found to show very good response to the phase transition in phospholipid vesicles. The anisotropy shows a sudden drop at $42^{\circ} \mathrm{C}$, the phase transition temperature of DPPC. Hence, it was chosen to monitor the effect of bile salt on phospholipid vesicles. The critical micelle concentrations (CMC) of the two bile salts $\mathrm{NaDC}$ and $\mathrm{NaC}$ in water $\left(25^{\circ} \mathrm{C}\right)$ were determined by using the fluorescent dye method employing DPH fluorescence intensity and anisotropy as tools. ${ }^{15,16}$ The CMCs thus found are $6 \mathrm{mM}$ for $\mathrm{NaDC}$ and $16 \mathrm{mM}$ for $\mathrm{NaC}$.

\subsection{Effect of low concentrations of $\mathrm{NaDC}$ and $\mathrm{NaC}$ on DPPC SUVS}

Figures $1 \mathrm{a}$ and $\mathrm{b}$ show the variation of fluorescence anisotropy of DPH with temperature in DPPC SUVs on addition of various concentrations of $\mathrm{NaDC}$ and $\mathrm{NaC}$, respectively. The concentration of bile salt was varied between $0 \cdot 1$ and $1 \mathrm{mM}$, keeping the DPPC concentration constant at $0.3 \mathrm{mM}$. Plots for a few intermediate concentrations have been removed from the figure for clarity.

There is no appreciable change in the anisotropy profiles of DPH observed in DPPC SUVs in presence of $\mathrm{NaC}$ in this concentration range, which clearly indicates that $\mathrm{NaC}$, when present at low concentration, does not have significant effect on the phase behaviour of DPPC SUVs. However, unlike $\mathrm{NaC}$, the effect of NaDC on DPPC SUVs is remarkable even at these low concentration ranges. Variation of fluorescence anisotropy shows behaviour similar to that in pure DPPC SUVs up to $0.55 \mathrm{mM}$ of $\mathrm{NaDC}$, i.e. the anisotropy is high at lower temperature and shows a drop at the phase transition and then levels off on further increase in temperature. However, there is an almost linear decrease in phase-transition temperature in the concentration range of $0.1 \mathrm{mM}$ to $0.55 \mathrm{mM}$ of $\mathrm{NaDC}$; below $0.1 \mathrm{mM}$ of $\mathrm{NaDC}$ the phase-transition temperature remains practically the same as that of pure DPPC SUVs $\left(42^{\circ} \mathrm{C}\right)$. The transition temperature decreases from $42^{\circ} \mathrm{C}$ in pure DPPC to $35^{\circ} \mathrm{C}$ by $0.55 \mathrm{mM}$ of
$\mathrm{NaDC}$ (figure 2). This indicates that up to $0.55 \mathrm{mM}$ of $\mathrm{NaDC}$, incorporation of deoxycholate into the DPPC bilayer causes a disturbance in the interactions among the chains resulting in a decrease in the temperature of the gel to liquid crystal transition. Thus, in the above concentration range $(0 \cdot 1-$ $0.55 \mathrm{mM}), \mathrm{NaDC}$ has a fluidising effect on DPPC SUVs; the bilayer structure of bile salt incorporated vesicles is still intact showing distinct phase behaviour.

In the concentration window 0.6 to $1.0 \mathrm{mM}$ of $\mathrm{NaDC}$, the anisotropy shows variation similar to that of a pure DPPC bilayer below the phase transition temperature, but above this temperature it increases with increasing temperature, which is similar to that in pure NaDC micelles. ${ }^{16}$ It is known in the literature that the partition coefficient of $\mathrm{NaDC}$ depends on the physical state of the lipid membrane. ${ }^{17}$ Thus, in the present case it can be argued that as the temperature approaches the phase transition temperature more fluid regions in the bilayers are likely to arise, which in turn increases the permeability of the

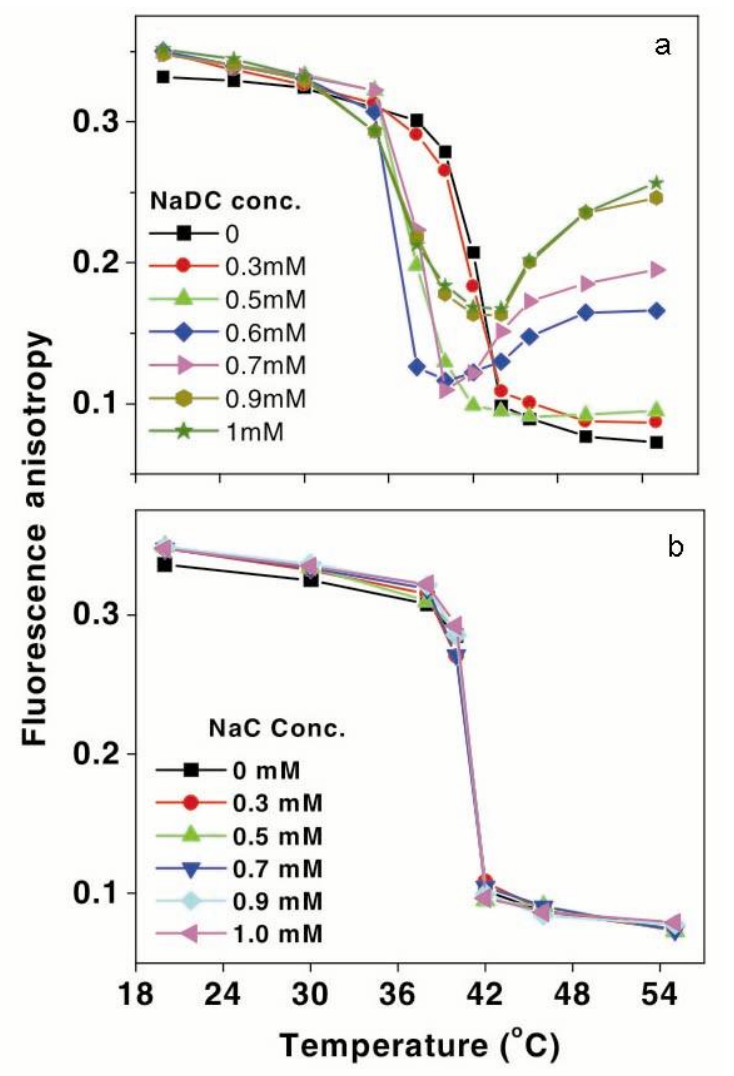

Figure 1. Response of DPH fluorescence anisotropy to the changes induced by (a) $\mathrm{NaDC}$ and (b) $\mathrm{NaC}(0-1 \mathrm{mM})$ in $0.3 \mathrm{mM}$ of DPPC SUVs $\left(\lambda_{\mathrm{ex}}=360 \mathrm{~nm}, \lambda_{\mathrm{em}}=428 \mathrm{~nm}\right)$. 
membrane towards NaDC so as to facilitate the formation of mixed micelles. Therefore, in the above concentration range of $\mathrm{NaDC}(0 \cdot 6-1 \mathrm{mM})$ there is coexistence of bile salt incorporated bilayers (at low temperature, i.e. below the phase transition temperature) and lipid-rich mixed micelles (at high temperature).

\subsection{Effect of high concentrations of NaDC on DPPC SUVS}

Figure 3 shows the fluorescence anisotropy profiles of DPH with temperature in DPPC SUVs in the presence of $\mathrm{NaDC}$ in the concentration range of $1.0 \mathrm{mM} \leqslant[\mathrm{NaDC}] \leqslant 6.0 \mathrm{mM}$. As the concentration of $\mathrm{NaDC}$ increases from 1.0 to $3.0 \mathrm{mM}$, the micellar character becomes prominent; however there still exists the signature of bilayer phase transition at lower temperature. By $4.0 \mathrm{mM}$ of $\mathrm{NaDC}$ the phase transition is completely abolished and the anisotropy profiles exactly resemble those of pure $\mathrm{NaDC}$ micelles. This indicates that above $4.0 \mathrm{mM}$ of $\mathrm{NaDC}$, which is still lower than its $\mathrm{CMC}$ in water $(6 \mathrm{mM})$, the bilayer signature is completely lost and there is existence of micelles suggesting the solubilisation of phospholipids. There are literature reports on decrease in critical micelle concentration of bile salt on addition of phosphatidylcholine. ${ }^{18,19}$

The solubilisation of lipid vesicles by detergents such as Triton- $\mathrm{X}$ is known to proceed through four stages: Stage I: mixed vesicle formation, stage II: coexistence of mixed vesicles and mixed bilayer

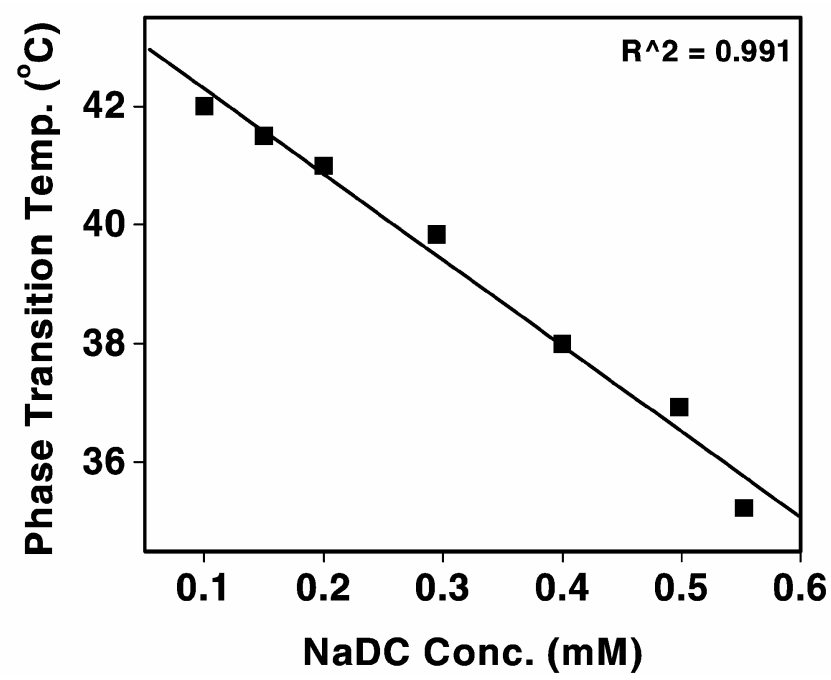

Figure 2. Variation of the main phase transition temperature of DPPC SUVs with NaDC concentration. sheets, stage III: transformation of mixed bilayers to lipid-rich micelles and stage IV: solubilisation of lipid in the detergent micelles. ${ }^{20}$ From our experimental data, a similar model shows the solubilisation of DPPC SUVs by NaDC. Thus, under experimental conditions, $0.3 \mathrm{mM}$ of DPPC SUVs, the different stages can be depicted as follows: (i) at $[\mathrm{NaDC}]<$ $0.6 \mathrm{mM}, \mathrm{NaDC}$ partitions into the bilayer and alters the chain order resulting in linear decrease in the phase transition temperature with $\mathrm{NaDC}$ concentration; (ii) at $0.6 \mathrm{mM} \leq[\mathrm{NaDC}]<4.0 \mathrm{mM}$, there is coexistence of mixed vesicles and micelles, below the phase transition the bilayer signature is observed and near the phase transition temperature the permeability of the bilayer for $\mathrm{NaDC}$ increases, thus more of $\mathrm{NaDC}$ get associated with the bilayer, converting them to mixed micelles; (iii) at $[\mathrm{NaDC}] \geq 4.0 \mathrm{mM}$, complete solubilisation of DPPC lipids in $\mathrm{NaDC}$ micelles takes place and there is no signature of bilayer observed.

This can be presented in a flow diagram as below:

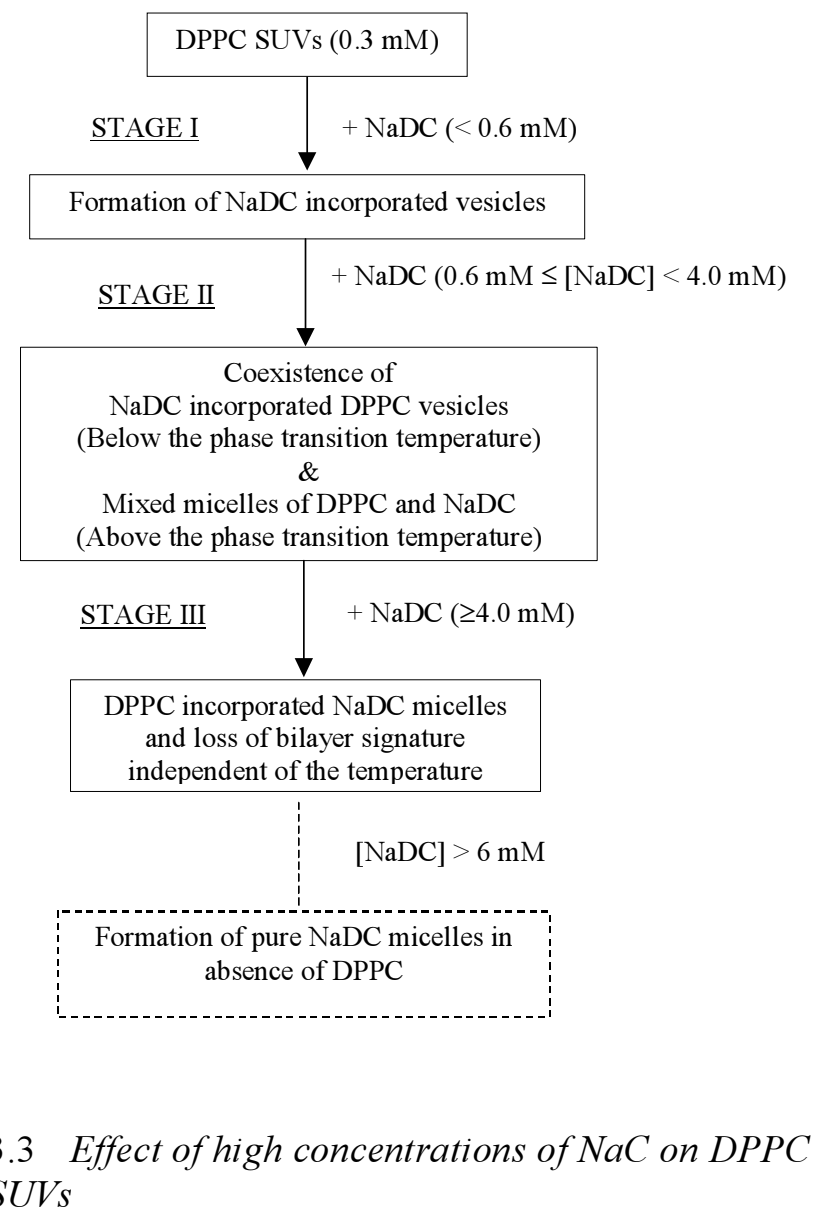

Figure 4 shows temperature-dependent anisotropy profiles of DPH in DPPC SUVs indicating the effect 
of $\mathrm{NaC}$ on DPPC SUVs in the higher concentration range. $\mathrm{NaC}$ concentration is varied from $4-20 \mathrm{mM}$. The bilayer behaviour is retained even up to a concentration of $14 \mathrm{mM}$ of $\mathrm{NaC}$. However, the phase transition temperature diminishes with increase in $\mathrm{NaC}$ concentration; it drops from $42^{\circ} \mathrm{C}$ in pure DPPC to $32^{\circ} \mathrm{C}$ in presence of $14 \mathrm{mM}$ of $\mathrm{NaC}$. Above $16 \mathrm{mM}$ of $\mathrm{NaC}$ the anisotropy profile shows similar trend as that of pure $\mathrm{NaC}$ micelles in the higher

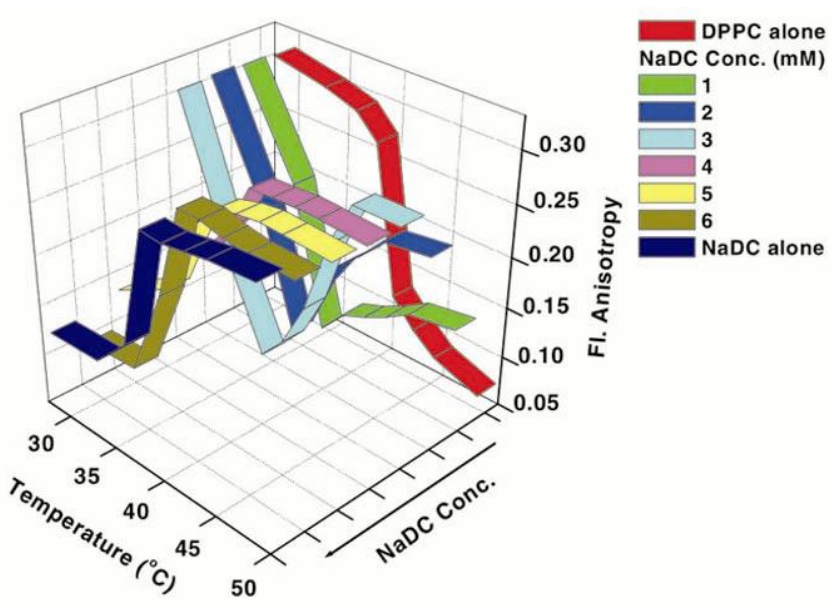

Figure 3. $3 \mathrm{D}$ plots showing variation of steady-state fluorescence anisotropy of DPH in DPPC SUVs as a function of temperature. [DPPC] $=0.3 \mathrm{mM},[\mathrm{NaDC}]=$ $1.0-6.0 \mathrm{mM}\left(\lambda_{\mathrm{ex}}=360 \mathrm{~nm}, \lambda_{\mathrm{em}}=428 \mathrm{~nm}\right)$. The variation of fluorescence anisotropy in pure $\mathrm{NaDC}$ micelles is also given for comparison.

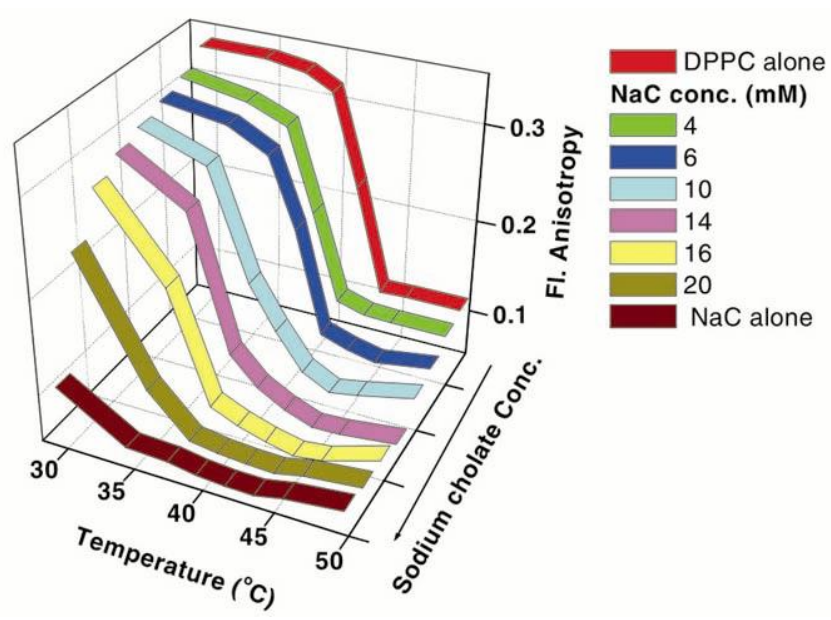

Figure 4. Temperature-dependent steady-state fluorescence anisotropy profiles of DPH in DPPC SUVs in presence of $\mathrm{NaC}$ at higher concentration range. [DPPC] = $0.3 \mathrm{mM}$. $[\mathrm{NaC}]=4.0-20 \mathrm{mM}\left(\lambda_{\mathrm{ex}}=360 \mathrm{~nm}, \lambda_{\mathrm{em}}=428 \mathrm{~nm}\right)$. The temperature dependence of anisotropy in pure $\mathrm{NaC}$ micelles is also given for comparison. temperature regime. In case of $\mathrm{NaC}$, the complete solubilisation of phospholipid vesicles takes place only when the concentration of $\mathrm{NaC}$ is above its CMC.

The efficiency of a bile salt towards membrane perturbation is found to be dependent on its nature. For efficient perturbance to occur at a concentration prior to the critical micelle concentration of the bile salt, it is expected that the affinity of the bile salt for the membrane should be high as compared to the affinity of the bile salt for itself, which is clearly the case for the lipophilic deoxycholate. $\mathrm{NaC}$ being more hydrophilic does not interact with the membrane efficiently and hence its solubilising capacity towards phospholipid vesicles is less.

\subsection{Experiments with different concentrations of DPPC SUVS}

It was found that the first appearance of mixed micelles occurs at $0.6 \mathrm{mM}$ of $\mathrm{NaDC}$ for $0.3 \mathrm{mM}$ of DPPC SUVs, which comes out to be a ratio of $1: 2$ of lipid to NaDC. In order to examine whether this ratio of lipid to $\mathrm{NaDC}$ is important for mixed micelle formation, experiments were carried out with different concentrations of DPPC SUVs and $\mathrm{NaDC}$ maintaining their ratio at $1: 2$.

Figure 5 shows the temperature-dependent fluorescence anisotropy profiles of DPH at different concentrations of DPPC SUVs and NaDC mixed in a fixed ratio of $1: 2$. It is clear from the figure that although the ratio of DPPC to NaDC is kept con-

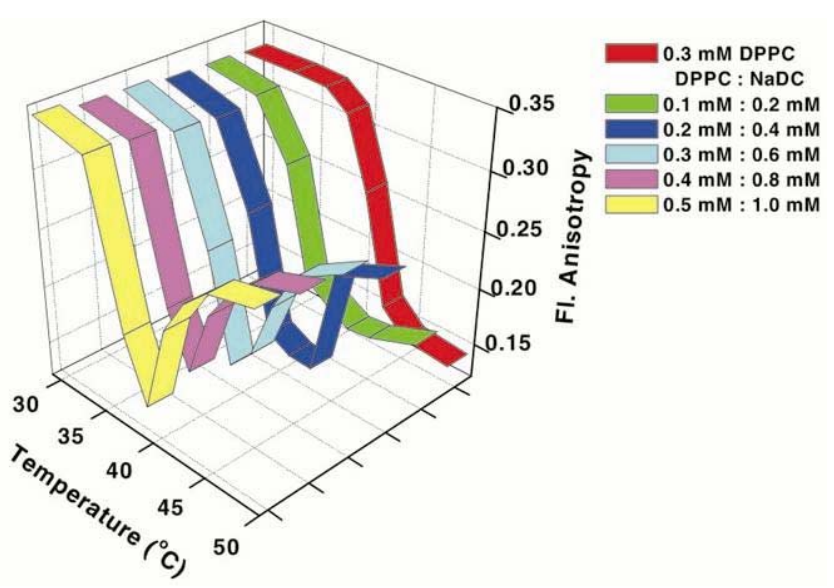

Figure 5. Temperature-dependent steady-state fluorescence anisotropy profiles of DPH in mixture of DPPC SUVs and NaDC at a ratio of $1: 2$. [DPPC] $=0 \cdot 1-0.5 \mathrm{mM}$ and $[\mathrm{NaDC}]=0 \cdot 2-1 \cdot 0 \mathrm{mM}\left(\lambda_{\mathrm{ex}}=360 \mathrm{~nm}, \lambda_{\mathrm{em}}=428 \mathrm{~nm}\right)$. 
stant, the anisotropy profiles differ from each other. This indicates that the formation of mixed micelles of phospholipid vesicles and bile salts is strongly dependent on the absolute concentrations of lipid and bile salt and not just on their molar ratio. This is in agreement with earlier literature reports that the effect of surfactant on bilayer depends on the "effective" molar ratio of detergent/phospholipid in the bilayer and not on the "total" molar ratio ${ }^{17}$.

\section{Conclusions}

The present study provides an insight into the mechanism of interaction of bile salts with small unilamellar vesicles. Based on the experimental findings it is possible to propose the concentration ranges involved with different stages of changes in the solubilisation of DPPC SUVs by NaDC. The experiments showed that the concentration of a bile salt needed to bring about a certain change in the vesicle is strongly dependent on the absolute concentrations of both phospholipid and a bile salt and not on their molar ratio. As compared to $\mathrm{NaDC}$, the effect of $\mathrm{NaC}$ on DPPC SUVs is found to be subtle. $\mathrm{NaC}$ when added at a concentration above its CMC $(16 \mathrm{mM})$ is found to solubilise the vesicles.

\section{References}

1. Blume A and Garidel P 1999 The handbook of thermal analysis and calorimetry, Vol. 4, From macromolecules to man (ed.) R B Kemp (Amsterdam: Elsevier) ch. 4, p. 109
2. Marsh D 1996 Handbook of nonmedical application of liposomes (eds) D Lasic and Y Barenholz (Boca Raton, FL: CRC Press) vol. 2, ch. 1, p. 1

3. Lichtenberg D 1993 Biomembranes - physical aspects (ed.) M Shinitzky (Weinheim: VCH) p. 63

4. Carey M C, Small D M and Bliss C M 1983 Аnnu. Rev. Physiol. 45651

5. Elorza M A, Elorza B and Chantres J R 1997 Int. J. Pharma. 158173

6. Schubert R, Wolburg H, Schmidt K H and Roth H J 1991 Chem. Phys. Lip. 58121

7. Coleman R 1987 Biochem. Soc. Trans. 15 S68

8. Hildebrand A, Neubert R, Garidel P and Blume A 2002 Langmuir 182836

9. Hildebrand A, Beyer K, Neubert R, Garidel P and Blume A 2003 Colloids Surf. B32 335

10. New R R C 1990 Liposomes, a practical approach (New York: Oxford University Press)

11. Santos N C, Prieto M and Castanho M A R B 2003 Biochim. Biophys. Acta 1612123

12. Shinitzky M and Barenholz Y 1978 Biochem. Biophys. Acta $\mathbf{5 1 5} 367$

13. Glatz P 1978 Anal. Biochem. 87187

14. Kaiser R D and London E 1998 Biochemistry 37 8180

15. Chattopadhyay A and London E 1984 Anal. Biochem. 139408

16. Subuddhi U 2006 Fluorescent molecular probes for phospholipid vesicles and bile salt micelles, $\mathrm{Ph} \mathrm{D}$ thesis, Indian Institute of Technology-Madras, Chennai

17. Chantres J R, Elorza B, Elorza M A and Rodado P 1996 Int. J. Pharm. 138139

18. Carey M C and Small D M 1972 Arch. Int. Med. 130 506

19. Small D M 1971 The bile acids - Chemistry, physiology and metabolism (eds) P P Nair and D Kritchevsky (New York: Plenum) vol. 1, pp 247-354

20. Lasch J 1995 Biochim. Biophys. Acta 1241269 